NSF-KITP-03-30

MCTP-03-19

hep-th/0305055

\title{
Comments on Supergravity Description of S-branes
}

\author{
Alex Buchel ${ }^{1}$ and Johannes Walcher ${ }^{2}$ \\ ${ }^{1}$ Michigan Center for Theoretical Physics \\ Randall Laboratory of Physics, The University of Michigan \\ Ann Arbor, MI 48109-1120, USA \\ ${ }^{2}$ Kavli Institute for Theoretical Physics \\ University of California \\ Santa Barbara, CA 93106, USA
}

\begin{abstract}
This is a note on the coupled supergravity-tachyon matter system, which has been earlier proposed as a candidate for the effective space-time description of S-branes. In particular, we study an ansatz with the maximal $I S O(p+1) \times S O(8-p, 1)$ symmetry, for general brane dimensionality $p$ and homogeneous brane distribution in transverse space $\rho_{\perp}$. A simple application of singularity theorems shows that (for $p \leq 7$ ) the most general solution with these symmetries is always singular. (This invalidates a recent claim in the literature.) We include a few general comments about the possibility of describing the decay of unstable D-branes in purely gravitational terms.
\end{abstract}

May 2003 


\section{Introduction}

Unstable D-branes and their tachyons are a rich source of interesting problems in string theory. While the kinematics of tachyon condensation and the relation to Dbrane charges is by now fairly well understood, the decay of unstable branes as a time-dependent process has attracted a considerable amount of attention only recently. Guided by intuition from ordinary stable D-branes, one is led to expect that this process has both a microscopic (or "open string") and a macroscopic (or "closed string") description, which might in some sense be "dual" to each other. In fact, it was advocated in [1] that the process of unstable brane creation and decay should be viewed as the direct spacelike analog of the familiar timelike branes. A recent selection of literature on the subject is [1-24].

An important part of the problem is the effective spacetime description of the decay process. Most of the candidate supergravity solutions for S-branes that have been written down so far do not satisfy the basic conditions on singularity type and global structure. This might be due to the restrictiveness of the ansatz used or to the fact that the proper set of relevant degrees of freedom has not been identified. A hint that the latter might actually be the case comes from the recent studies of open string theory with time-dependent boundary perturbations $[2,14,16,17]$. These results lead to the reasonable question whether the decay of unstable branes does actually admit a decoupling limit that would be the necessary requirement for simplifications. See the end of this note for some additional comments on these issues.

It was proposed in [11] that a clue to resolving or understanding the singularities in the spacetime description of S-branes might lie in including the open string tachyon explicitly in the dynamics, coupling the tachyon to (super)gravity via the Dirac-BornInfeld type of action commonly known as tachyon matter. To avoid confusion, we note that this approach is not equivalent to studying unstable D-brane probes in the background geometry of $[1,3,4]$. In fact, the main point of [11] was to view the asymptotic geometry of $[1,3,4]$ as the result of the full gravitational backreaction of the time-dependent decay process of (a large number of) unstable D-branes. Translated to say the time-like D-branes in type IIB string theory, the question analogous to the one raised in [11] would be how to reconstruct the classical $p$-brane solution in supergravity from the corresponding boundary state [26].

The toy model discussed in [11] is 4-dimensional Einstein-Maxwell theory coupled 
to tachyon matter on a distribution of one-dimensional defects ("D1-branes"). In this toy model, it was shown that all solutions are generically singular. In particular, it was shown that including the tachyon matter generically destabilizes the horizon, turning it into a spacelike (or null) curvature singularity. Moreover, it was shown that including the tachyon matter does not remove the time-like singularities found previously. Again, we emphasize that these statements were based on an analysis that accounted for the full backreaction of the tachyon matter on the geometry. The most significant drawback of the approach of [11] was the requirement of $I S O(1) \times S O(2,1)$ symmetry. This symmetry, which is the maximal possible symmetry that one could expect for an S-brane of this type, is expected to be broken in the real situation. In particular, including tachyon matter would break this symmetry unless the branes are smeared uniformly in the transverse space. How to properly reduce this symmetry requirement is an important problem.

Another question is what happens in 10 dimensions and for general brane dimensionality $p$. It was argued in [11] that the qualitative behavior should be the same as in the toy model, but no explicit computations were performed to substantiate this claim. Recent results presented by Leblond and Peet in [13] appear to show that the higher-dimensional analogues of [11] do admit completely non-singular solutions. Triggered by these results, our initial motivation for the present work was to verify this possibility. However ${ }^{1}$, we will here show that non-singular solutions of this system can in fact be easily excluded in general, thus confirming the behavior anticipated in [11]. These results follow from the application of a simple singularity theorem based on the strong energy condition satisfied by the tachyon matter, for $p_{(=)}^{<} 7$. (The case $p=8$ is special and we are unable to exclude non-singular solutions on general grounds.) While at first sight these results might seem discouraging, we wish to emphasize that we do share the hope that tachyon condensation as a dynamical process will ultimately admit a complete and physically reasonable description.

\section{Tachyon matter coupled to supergravity}

\subsection{Action and equations of motion}

In this note, we study a system of supergravity fields $S_{\text {bulk }}$ coupled to a (DBI+WZ)type Lagrangian $S_{\text {brane }}$ known as tachyon matter. We think of $S_{\text {brane }}$ as representing the

\footnotetext{
${ }^{1}$ The discrepancy of [13] seems to have both an analytical and a numerical origin. See the appendix and the forthcoming publication [29] for comments.
} 
degrees of freedom of an unstable D-brane system that backreacts on the geometry. In this section, we will write out the equations of motion in Einstein frame in a convenient form that will make the appearance of singularities most obvious. Completely explicit formulas, as well as the translation to the string frame, are relegated to the appendix. The full action for the coupled system is (the brane has $p+1$ spatial dimensions)

$$
\begin{aligned}
S= & S_{\text {bulk }}+S_{\text {brane }} \\
= & \frac{1}{16 \pi G_{10}} \int d^{10} x \sqrt{-g}\left(R-\frac{1}{2}(\partial \phi)^{2}-\frac{e^{a \phi}}{2(p+2) !} F_{p+2}^{2}\right) \\
& +\frac{\Lambda}{16 \pi G_{10}} \int d^{p+2} x_{\|} \varrho_{\perp}\left(-V(T) \sqrt{-A} e^{-\phi}\right)+\frac{\Lambda}{16 \pi G_{10}} \int \varrho_{\perp} f(T) d T \wedge C_{p+1},
\end{aligned}
$$

where $a \equiv(3-p) / 2$ and

$$
A_{\mu \nu}=g_{\mu \nu} e^{\phi / 2}+\partial_{\mu} T \partial_{\nu} T
$$

Here and below we use the symbol $\|$, and Greek indices $\mu, \nu, \alpha, \beta, \ldots$ for the directions along the unstable brane (including time) and the symbol $\perp$ or Roman indices $i, j, \ldots$ for the transverse directions ${ }^{2}$. Capital Roman indices $M, N, \ldots$ will denote all directions together.

In (1), $\varrho_{\perp}$ describes the distribution of branes in the transverse directions. Let us comment. As written in (1), $\varrho_{\perp}$ is a form of degree $8-p$, proportional to the (appropriately normalized) volume form of the transverse space,

$$
\varrho_{\perp}=\rho_{\perp} d^{8-p} x_{\perp}
$$

The density $\rho_{\perp}$ (transforming with the determinant of the Jacobian) can depend on the transverse directions, but it is independent of the $x_{\|}$. Below, we will make an $S O(p+1) \times S O(8-p, 1)$ symmetric ansatz, which requires $\rho_{\perp}$ to satisfy eq. $(10) .{ }^{3}$

As already reviewed in [11], the couplings $V(T)$ and $f(T)$ are not known precisely. In [2] it was argued that $V(T)>0$, and

$$
V(T) \propto e^{-\alpha|T| / 2}, \quad \text { as } \quad|T| \rightarrow \infty,
$$

\footnotetext{
${ }^{2}$ We are assuming here that our spacetime is a direct product.

${ }^{3}$ Since we allow for warping of the transverse directions, the physical density of branes (branes per unit volume) is given by $\rho_{\text {phys }}$ in

$$
\varrho_{\perp}=\rho_{\text {phys }} \operatorname{vol}_{\perp}=\rho_{\text {phys }} \sqrt{g_{\perp}} d^{8-p} x_{\perp},
$$

where $\sqrt{g_{\perp}}$ depends on the parallel directions (below, it will only depend on time). The equation of motion for $\rho_{\text {phys }}$ is "free streaming" in the transverse directions.
} 
with $\alpha=\sqrt{2}$ for superstrings. For numerical analysis of the tachyon condensation a convenient choice is $V(T)=1 / \cosh (T / \sqrt{2})$, as was used in [11], and as recently derived in [24]. It was noted in [11] that the singularity argument for the S0-brane was robust as to the precise choice of $V(T), f(T)$. Here, we will again find that the precise expressions for $V(T), f(T)$ are not important. What matters is that $V(T)>0$ and vanishes only as $|T| \rightarrow \infty$.

The dilaton, flux, and tachyon equations of motion derived from (1) read, respectively,

$$
\begin{aligned}
& 0=\nabla^{2} \phi+\frac{\Lambda \rho_{\perp} e^{-\phi} V \sqrt{-A}}{\sqrt{-g}}\left(1-\frac{1}{4}\left(A^{-1}\right)^{\mu \nu} g_{\mu \nu} e^{\phi / 2}\right)-\frac{a}{2(p+2) !} e^{a \phi} F_{p+2}^{2} \\
& 0=\frac{1}{(p+1) !} \epsilon_{\mu \lambda_{2} \cdots \lambda_{p+2}} \partial_{\nu}\left(\sqrt{-g} e^{a \phi} F^{\nu \lambda_{2} \cdots \lambda_{p+2}}\right)+\Lambda \rho_{\perp} f \partial_{\mu} T \\
& 0=\epsilon_{\mu \lambda_{2} \cdots \lambda_{p+2}}\left[\partial_{\nu}\left(\Lambda \rho_{\perp} V e^{-\phi} \sqrt{-A}\left(A^{-1}\right)^{\nu \kappa} \partial_{\kappa} T\right)-\Lambda \rho_{\perp} \sqrt{-A} e^{-\phi} \frac{d V}{d T}\right]-\Lambda \rho_{\perp} f F_{\mu \lambda_{2} \cdots \lambda_{p+2}}
\end{aligned}
$$

Additionally, we have the Einstein equations

$$
R_{M N}=T_{M N}^{(1)}+T_{M N}^{(p+2)}+T_{M N}^{\text {brane }}
$$

where $T_{M N}$ denotes the trace reversed energy-momentum tensor of the dilaton, form field and tachyon matter, respectively. Explicitly,

$$
\begin{aligned}
T_{M N}^{(1)} & =\frac{1}{2} \partial_{M} \phi \partial_{N} \phi \\
T_{M N}^{(p+2)} & =\frac{e^{a \phi}}{2(p+1) !}\left(F_{M \ldots} F_{N} \cdots-\frac{(p+1)}{8(p+2)} F_{p+2}^{2} g_{M N}\right) \\
T_{\mu \nu}^{\text {brane }} & =\frac{\Lambda \rho_{\perp} V e^{-\phi / 2} \sqrt{-A}}{16 \sqrt{-g}}\left(\left(A^{-1}\right)^{\alpha \beta} g_{\alpha \beta} g_{\mu \nu}-8\left(A^{-1}\right)^{\alpha \beta} g_{\alpha \mu} g_{\beta \nu}\right) \\
T_{i j}^{\text {brane }} & =\frac{\Lambda \rho_{\perp} V e^{-\phi / 2} \sqrt{-A}}{16 \sqrt{-g}}\left(\left(A^{-1}\right)^{\alpha \beta} g_{\alpha \beta} g_{i j}\right),
\end{aligned}
$$

where dots in the flux stress tensor denote contraction of indices.

\subsection{A homogeneous cosmology}

As in [11], we will impose the maximal possible symmetry on our system. As explained in [1] (see also $[3,4,11]$ ) this maximal symmetry is $I S O(p+1) \times S O(8-p, 1)$. Thus, the parallel space is flat $(p+1)$-dimensional Euclidean space, and the transverse space 
is the hyperbolic space $H_{8-p}$. But to make contact with [3,4], and also [13], we will write out the equations in a slightly more general form, allowing arbitrary constant curvature $k_{\|}$and $k_{\perp}$ in both the parallel and transverse directions. The metric ansatz can then be written in the form

$$
d s^{2}=-d t^{2}+a_{\|}(t)^{2} d x_{\|}^{2}+a_{\perp}(t)^{2} d x_{\perp}^{2}
$$

The ansatz for the flux, dilaton, and tachyon is quite simply

$$
F_{t, x_{1}, \cdots, x_{p+1}}=A(t) a_{\|}^{p+1}, \quad \phi \equiv \phi(t), \quad T \equiv T(t)
$$

We also need to supply the distribution of branes in the transverse space. As already indicated, only a homogeneous distribution is consistent with the symmetries and simple enough to allow an analytic treatment. For instance, if transverse space is $H_{8-p}$, we set

$$
\rho_{\perp}=\rho_{0} \sqrt{g_{H_{8-p}}},
$$

where $g_{H_{8-p}}$ is the determinant of $d H_{8-p}^{2}$, and $\rho_{0}$ is a constant. Despite this high amount of symmetry, we expect that some of our statements are actually more general.

The ansatz (8) is simply a ten-dimensional homogeneous, but non-isotropic FRW cosmology. As is well-known, this type of cosmology is not generically non-singular, and has at least either a Big Bang or a Big Crunch singularity. This knowledge is backed by powerful singularity theorems, see, e.g., [25]. Therefore, if the goal is to construct non-singular solutions, one has to make sure that one uses exotic forms of matter that allow evading the singularity theorems. We will now show that the tachyon matter is, in fact, not quit exotic enough.

Our action (1) contains three types of matter fields, the tachyon, dilaton and RRform field. The explicit expressions for their energy density, $\rho$, and parallel and transverse pressure, $P_{\|}$and $P_{\perp}$, are given in table 1 . Let us also introduce the parallel and transverse Hubble parameters, $H_{\|}=a_{\|} / a_{\|}$and $H_{\perp}=a_{\perp} / a_{\perp}$. With this notation, the equations of motion for the ansatz (8) are

$$
\begin{aligned}
-(p+1) \frac{\ddot{a_{\|}}}{a_{\|}}-(8-p) \frac{\ddot{a_{\perp}}}{a_{\perp}} & =\frac{1}{8}\left(7 \rho+(p+1) P_{\|}+(8-p) P_{\perp}\right) \\
\frac{\ddot{a_{\|}}}{a_{\|}}+p\left(H_{\|}^{2}+\frac{k_{\|}}{a_{\|}^{2}}\right)+(8-p) H_{\perp} H_{\|} & =\frac{1}{8}\left(\rho+(7-p) P_{\|}-(8-p) P_{\perp}\right) \\
\frac{\ddot{a}_{\perp}}{a_{\perp}}+(7-p)\left(H_{\perp}^{2}+\frac{k_{\perp}}{a_{\perp}^{2}}\right)+(p+1) H_{\|} H_{\perp} & =\frac{1}{8}\left(\rho+p P_{\perp}-(p+1) P_{\|}\right) .
\end{aligned}
$$




\begin{tabular}{|l|c|c|c|}
\hline & $\rho$ & $P_{\|}$ & $P_{\perp}$ \\
\hline Tachyon & $\frac{\lambda V(T) e^{\phi(p / 4-1 / 2)}}{2 a_{\perp}^{8-p} \sqrt{\Delta}}$ & $-\rho \Delta=-\frac{\lambda V(T) e^{\phi(p / 4-1 / 2)} \sqrt{\Delta}}{2 a_{\perp}^{8-p}}$ & 0 \\
Dilaton & $\frac{1}{4} \dot{\phi}^{2}$ & $\frac{1}{4} \dot{\phi}^{2}$ & $\frac{1}{4} \dot{\phi}^{2}$ \\
RR form & $\frac{1}{4} e^{a \phi} A^{2}$ & $-\frac{1}{4} e^{a \phi} A^{2}$ & $\frac{1}{4} e^{a \phi} A^{2}$ \\
\hline
\end{tabular}

Table 1: Energy density and pressure (31) for the three matter fields in (1) in the cosmology (8). Here, $\Delta=1-e^{-\phi / 2} \dot{T}^{2}$, and $\lambda=\rho_{0} \Lambda$.

As is familiar, the equations (1) are not independent. The constraint (Friedmann equation) is

$$
\frac{p(p+1)}{2}\left(H_{\|}^{2}+\frac{k_{\|}}{a_{\|}^{2}}\right)+\frac{(7-p)(8-p)}{2}\left(H_{\perp}^{2}+\frac{k_{\perp}}{a_{\perp}^{2}}\right)+(p+1)(8-p) H_{\|} H_{\perp}=\rho
$$

and is consistent with the equations of motion (11) precisely if the energy is covariantly conserved

$$
\dot{\rho}=-(p+1) H_{\|}\left(\rho+P_{\|}\right)-(8-p) H_{\perp}\left(\rho+P_{\perp}\right) .
$$

To check this, we record here the equations for the energy density and pressure that follow from the corresponding equations of motion (eq. (29) in the appendix is helpful for this). We have for the tachyon

$$
\dot{\rho}^{\text {tach }}=-(p+1) H_{\|}\left(\rho^{\text {tach }}+P_{\|}^{\text {tach }}\right)-(8-p) H_{\perp} \rho^{\text {tach }}-\frac{\lambda A f}{2 a_{\perp}^{8-p}} \dot{T}+\frac{\dot{\phi} \rho^{\text {tach }}}{4}+(3-p) \frac{\dot{\phi} P_{\|}^{\text {tach }}}{4} .
$$

The first two terms are of course nothing but the covariant derivative of the energymomentum tensor, while the other terms describe energy exchange with the other matter fields. For the dilaton the corresponding equation is

$$
\dot{\rho}^{\mathrm{dil}}=-\left((p+1) H_{\|}+(8-p) H_{\perp}\right)\left(\rho^{\mathrm{dil}}+P^{\mathrm{dil}}\right)+a \rho^{\mathrm{RR}} \dot{\phi}-\frac{\dot{\phi} \rho^{\mathrm{tach}}}{4}-(3-p) \frac{\dot{\phi} P_{\|}^{\mathrm{tach}}}{4},
$$

while for the form field we have

$$
\dot{\rho}^{\mathrm{RR}}=-(8-p) H_{\perp}\left(\rho^{\mathrm{RR}}+P_{\perp}^{\mathrm{RR}}\right)+\frac{\lambda A f}{2 a_{\perp}^{8-p}} \dot{T}-a \rho^{\mathrm{RR}} \dot{\phi} .
$$

The consistency condition (13) follows trivially by taking the sum of (14)-(16). 


\subsection{A singularity theorem $(p<7)$}

We wish to show that solutions of (11) are generically singular. For a textbook argument, consider the average warp factor $a$, defined by

$$
a^{9}=a_{\|}^{p+1} a_{\perp}^{8-p} .
$$

We have

$$
9 \frac{\dot{a}}{a}=9 H=(p+1) H_{\|}+(8-p) H_{\perp},
$$

and using (11), we find,

$$
\begin{aligned}
9 \frac{\ddot{a}}{a} & =(p+1) \frac{\ddot{a_{\|}}}{a_{\|}}+(8-p) \frac{\ddot{a_{\perp}}}{a_{\perp}}-\frac{(p+1)(8-p)}{9}\left(H_{\|}-H_{\perp}\right)^{2} \\
& =-\frac{1}{8}\left(7 \rho+(p+1) P_{\|}+(8-p) P_{\perp}\right)-\frac{(p+1)(8-p)}{9}\left(H_{\|}-H_{\perp}\right)^{2},
\end{aligned}
$$

Now from table 1 , one easily deduces that the combination $7 \rho+(p+1) P_{\|}+(8-p) P_{\perp}$ on the right hand side of (19) is always positive for $p<7$. This is a strong energy condition, and it implies that $\ddot{a}$ will be always be negative. Thus, if we consider a contracting phase of the universe with $H<0,{ }^{4}$ the slope of $a$ is also negative and cannot increase. This implies that $a$ will reach zero in a finite time bounded above by $1 /|H|$. Intuitively, this contraction of the universe will lead to a diverging energy density, hence one expects a curvature singularity. While this is not immediate from what we have said so far, and there could still be a continuation beyond the point where $a=0$, at the very least, the coordinate system (8) will not cover all of spacetime.

\subsection{More careful arguments $(p<7)$}

First of all let us note that asymptotically flat initial condition as $t \rightarrow-\infty$ for the S-brane cosmology implies that $H(t)<0$ as $t \rightarrow-\infty$. Indeed, asymptotic flatness of the $I S O(p+1) \times S O(8-p, 1)$ symmetry preserving ansatz $(8)$ implies

$$
\begin{array}{ll}
\left.a_{\|}\right|_{t \rightarrow-\infty} \rightarrow \text { constant, } & \left.a_{\|}\right|_{t \rightarrow-\infty} \sim o\left(\frac{1}{t}\right), \\
\left.a_{\perp}\right|_{t \rightarrow-\infty} \rightarrow-t, & \left.a_{\perp}\right|_{t \rightarrow-\infty} \rightarrow-1,
\end{array}
$$

thus $H$ as defined by (18) satisfies

$$
\left.H\right|_{t \rightarrow-\infty}=\frac{8-p}{9 t}+o\left(\frac{1}{t}\right)<0 .
$$

\footnotetext{
${ }^{4}$ The case $H>0$ is similar, with a singularity in the past.
} 
Moreover, from (19), we deduce

$$
\dot{H}+H^{2} \leq 0
$$

and as a consequence, we obtain the estimate

$$
H \leq \frac{1}{t-t_{s}}
$$

as long as $t$ is less that the integration constant $t_{s}$. Thus we conclude that $H$ will diverge at some finite time $t=t_{s}$,

$$
\left.H\right|_{t \rightarrow t_{s}} \rightarrow-\infty
$$

The arguments we have given so far do not imply that there is an actual physical singularity at $t=t_{s}$. The simplest way to show that this divergence is not just a signal of breakdown of the coordinate system (8) would be to establish that the Ricci scalar (or any other scalar curvature invariant) diverges as one approaches $t=t_{s}$. While we expect this to be the case, we have not been able to find a convincing argument. It is possible that while the universe is overall contracting, it is expanding in one of the two directions at the approach of $t=t_{s}$. This expansion could dilute the energy density so much as to prevent a curvature singularity. One would then hope that this is just a coordinate (e.g., Milne) singularity and that the spacetime admits an extension and a continuation beyond $t=t_{s}$. One desirable feature of this would be that the additional parts of spacetime are not in the Cauchy development of the $t \rightarrow-\infty$ asymptotic infinity, as one would expect for an S-brane. But even if this were the case, it appears that the timelike geodesics associated with energy flow of our matter are necessarily incomplete [25]. Additional input would be needed to make sense of the singularity.

We also note that our assumptions for proving the existence of this singularity were rather weak, and that, in particular, we did not assume invariance under time reversal $t \leftrightarrow-t$. Since the arguments only rely on the strong energy condition, which is satisfied for $p<7$, it is reasonable to expect that similar statements about singularities will hold even without assuming any particular symmetry.

\section{$2.5 p=7$ and $p=8$ cases}

Even though the strong energy condition does not hold for $p=7$, this case can be treated in essentially the same way $^{5}$ as in the previous subsection. The technical details are discussed in the appendix.

\footnotetext{
${ }^{5}$ Of course one should find a (new) relevant "singularity theorem".
} 
The $p=8$ case is special: this $\mathrm{S}$-brane is realized as the gravitational backreaction of the time-dependent decay of the unstable D9-brane. Since the D9-brane is space filling, there is no transverse space, and thus brane smearing (10) is irrelevant. One is then dealing with a ten-dimensional homogeneous and isotropic flat FRW cosmology. The tachyon matter close to the top of the tachyon potential and also the RR-form act effectively like a positive cosmological constant with $\rho=-P_{\|}=-P>0$. However, since $k_{\|}=0$, one cannot reasonably hope for a deSitter-like bounce. The best one can expect is an infinite period of inflation if tachyon matter and RR-form dominate over the dilaton for early/late times (this could be viewed as a "half S-brane"). We have investigated this question numerically, but have been unable to identify a solution of this kind. What seems to happen is that the dilaton is always activated so rapidly that its positive pressure induces the collapse of spacetime. Analytically, we have been able to exclude bouncing solutions that are invariant under time reversal, see eq. (33) in the appendix.

\section{Final Comments}

We have seen that the general time-dependent solution of the coupled supergravitytachyon matter system with maximal symmetries is singular. This confirms the expectations that the toy model considered in [11] in fact did capture most of the essential physics of the problem. One might ask the question whether this statement would still hold true if one were to relax the requirements of symmetry. Here one can imagine breaking the maximal $I S O(p+1) \times S O(8-p, 1)$ symmetry either to $I S O(p+1) \times S O(8-p)$ (by removing the smearing), or even further by breaking the $I S O(p+1)$ S-brane world volume symmetry, for instance by allowing more generic, spatially inhomogeneous timedependent profiles of the tachyon field on the unstable D-brane (see, e.g., [23] for a worldsheet approach to this situation). It is possible that since the arguments of the singularity theorem presented here (in the context of the maximally symmetric case) rely on the strong energy condition for the supergravity and tachyon matter in the decay of the unstable brane, the spatially inhomogeneous decay would still describe a singular cosmology in the supergravity approximation.

We conclude this note with a few incomplete comments about the possibility of describing the decay process of unstable D-branes in a classical supergravity theory. The first observation that it is really the supergravity approximation that is at fault in 
generating the singularities comes from the origin of the singularity in the toy model of [11]. In [11] the space-like singularity of the S0-brane was attributed to the divergence of the tachyon matter energy density (but not to the infinite energy ${ }^{6}$ from smearing). Furthermore, this energy density diverged precisely because the time derivative of the tachyon field was getting very large

$$
\dot{T} \propto \frac{1}{\sqrt{t-t_{s}}} \quad \text { as } \quad t \rightarrow t_{s}+0,
$$

that is the tachyon was "rolling too fast". But large gradients imply that the DBI approximation $^{7}$, used to couple open string tachyon to the closed string background in (1), is invalid at times $t \lesssim t_{s}$. In other words, as the tachyon approaches the top of the potential, the higher derivatives of the tachyon field in the effective action become more and more important. The latter indicates that massive string modes might not decouple.

A possibly related observation was made in $[14,16]$, in which the quantum open string creation in Sen's rolling tachyon background (timelike Liouville theory) was computed, and found to diverge due to the exponentially growing density of massive open string states. This shows that the tachyon should roll much faster at initial times than deduced from the tachyon matter action. These results also seem to point to the incompleteness of the open string description and to the importance of closed strings.

The closed string couplings of the rolling tachyon worldsheets were studied in $[2$, 18-20]. Most recently, it was shown in [20] that the total amount of closed string radiation is finite in a bosonic string theory, at least for large $p$. This is intriguing, as it suggests that an unstable D-brane does not quite manage to completely decay into closed strings at the linearized level. One would again be led to the conclusion that some mysterious form of tachyon matter must intervene as the final state of tachyon condensation. The results of [20] also indicate that it is the massive closed strings that carry away most of the energy.

The computation of [20] was done for a single unstable D-brane. Restoring the factor of $N$ if there are $N$ unstable branes shows that the total amount of closed string radiation behaves as $N^{2}$, so that the ratio of radiated energy to initially present brane

\footnotetext{
${ }^{6}$ Note the energy density associated with the unstable brane distribution is finite.

${ }^{7}$ The derivation of tachyon matter effective action in [24] is valid only for slowly varying tachyon profiles.
} 
tension is given by

$$
\# \frac{N^{2}}{N / g_{s}}=\# g_{s} N
$$

where \# is a number of order 1 . Thus, if $g_{s} N$ is large, the radiated energy computed in the linearized approximation will always exceed the initially present energy, meaning that the backreaction on the tachyon has to be taken into account. Moreover, the results of [18] show that the coupling to massive closed string modes, while exponentially growing at late times, is actually small at early times. Therefore, these results do not exclude the possibility that there is a limit in which only the first few low-lying closed string modes are excited, very early on in the decay process. Given (26), the limit in question appears to be the usual Maldacena-type limit of large $g_{s} N$.

A very important issue ${ }^{8}$ is whether the decay of an unstable D-brane leads to a classical final state at all or whether the decay process is inherently quantum mechanical, possibly with a thermal final state. For instance, the decay of an unstable D-brane might liberate so much energy that a black hole is formed before this energy can escape to infinity, in particular in the large $g_{s} N$ limit. The recent computations of [17] should help settling these issues.

In any case, we currently feel that the most promising avenue for making progress on the supergravity description of S-branes (if it exists) is to relax the symmetry requirements. It particular, it would be interesting to improve/generalize the singularity analysis presented in this note to the problem of the spatially inhomogeneous decay of unstable D-branes [28].

Acknowledgments We would like to thank Gary Horowitz, Finn Larsen, Alex Maloney, Joe Polchinski, Arkady Tseytlin, and Henry Tye for useful discussions. We are grateful to Frederic Leblond and Amanda Peet for important communications. J.W. would like to thank the Michigan CTP for hospitality during the workshop on timedependent backgrounds, where the initial questions addressed in this note were raised. The work of A.B. is supported in part by the U.S. Department of Energy. The work of J.W. is supported in part by the NSF under Grant No. PHY99-07949.

\footnotetext{
${ }^{8}$ We thank Joe Polchinski for useful discussions on this. Similar points have been made by Martin
} Kruczenski. 


\section{A Appendix}

\section{A.1 Explicit equations}

Here we spell out a few more details of the equations of motion (5) and (6). In a slightly more general gauge than (8), the ansatz reads

$$
\begin{aligned}
& d s_{E}^{2}=-c_{1}(t)^{2} d t^{2}+c_{2}(t)^{2} d x_{p+1}^{2}+c_{3}(t)^{2} d H_{8-p}^{2} \\
& F_{t, x_{1}, \cdots, x_{p+1}}=A(t) c_{1} c_{2}^{p+1} \\
& \phi \equiv \phi(t), \quad T \equiv T(t) .
\end{aligned}
$$

Again, we introduce

$$
\Delta=1-e^{-\phi / 2} c_{1}^{-2}\left(T^{\prime}\right)^{2}, \quad \lambda \equiv \rho_{0} \Lambda
$$

and explicitly evaluate the equations ${ }^{9}$

$$
\begin{aligned}
& \left(e^{a \phi} c_{3}^{8-p} A\right)^{\prime}=\lambda f T^{\prime} \\
& \frac{1}{c_{1} c_{2}^{p+1} c_{3}^{8-p}}\left(c_{1}^{-1} c_{2}^{p+1} c_{3}^{8-p} \phi^{\prime}\right)^{\prime}=\frac{1}{2} a e^{a \phi} A^{2}+\frac{\lambda V e^{\phi(p / 4-1 / 2)}}{4 c_{3}^{8-p}}\left((3-p) \Delta^{1 / 2}-\Delta^{-1 / 2}\right) \\
& 0=c_{1} c_{2}^{p+1}\left(\frac{d V}{d T} e^{\phi(p / 4-1 / 2)} \Delta^{1 / 2}+A f\right)+\left(c_{1}^{-1} c_{2}^{p+1} V e^{\phi(p / 4-1)} \Delta^{-1 / 2} T^{\prime}\right)^{\prime} .
\end{aligned}
$$

For the Einstein equations we have the nontrivial Ricci components

$$
\begin{aligned}
c_{1}^{-2} R_{t t} & =-\frac{p+1}{c_{1} c_{2}}\left(\frac{c_{2}^{\prime}}{c_{1}}\right)^{\prime}-\frac{8-p}{c_{1} c_{3}}\left(\frac{c_{3}^{\prime}}{c_{1}}\right)^{\prime} \\
c_{2}^{-2} R_{\mu \mu} & =\frac{1}{c_{1} c_{2}}\left(\frac{c_{2}^{\prime}}{c_{1}}\right)^{\prime}+\frac{p}{c_{2}^{2}}\left(\frac{c_{2}^{\prime}}{c_{1}}\right)^{2}+\frac{8-p}{c_{1}^{2}}\left(\frac{c_{2}^{\prime}}{c_{2}}\right)\left(\frac{c_{3}^{\prime}}{c_{3}}\right) \\
c_{3}^{-2} R_{i i} & =\frac{1}{c_{1} c_{3}}\left(\frac{c_{3}^{\prime}}{c_{1}}\right)^{\prime}+\frac{7-p}{c_{3}^{2}}\left(\left(\frac{c_{3}^{\prime}}{c_{1}}\right)^{2}-1\right)+\frac{p+1}{c_{1}^{2}}\left(\frac{c_{2}^{\prime}}{c_{2}}\right)\left(\frac{c_{3}^{\prime}}{c_{3}}\right),
\end{aligned}
$$

and the stress tensor

$$
\begin{aligned}
c_{1}^{-2} T_{t t} & =\frac{1}{2}\left(\frac{\phi^{\prime}}{c_{1}}\right)^{2}+\frac{e^{a \phi}(7-p)}{16} A^{2}+\frac{\lambda V e^{\phi(p / 4-1 / 2)}}{16 c_{3}^{8-p}}\left(7 \Delta^{-1 / 2}-(p+1) \Delta^{1 / 2}\right) \\
c_{2}^{-2} T_{\mu \mu} & =-\frac{e^{a \phi}(7-p)}{16} A^{2}+\frac{\lambda V e^{\phi(p / 4-1 / 2)}}{16 c_{3}^{8-p}}\left(\Delta^{-1 / 2}+(p-7) \Delta^{1 / 2}\right) \\
c_{3}^{-2} T_{i i} & =\frac{e^{a \phi}(1+p)}{16} A^{2}+\frac{\lambda V e^{\phi(p / 4-1 / 2)}}{16 c_{3}^{8-p}}\left(\Delta^{-1 / 2}+(p+1) \Delta^{1 / 2}\right) .
\end{aligned}
$$

\footnotetext{
${ }^{9}$ Prime denotes derivative with respect to $t$.
} 
The full system of equations is overdetermined (there is a standard first order constraint analogous to the Friedmann equation (12)), and we have explicitly verified that the complete system is consistent.

The "natural" S-brane solution in supergravity would be invariant under the timereversal $t \leftrightarrow-t$, with the tachyon sitting at the top of the potential at $t=0$. This implies that at $t=0$ we would like to have

$$
\phi^{\prime}=A=c_{2}^{\prime}=c_{3}^{\prime}=0 .
$$

The same boundary conditions were claimed in [11] to be inconsistent for describing the S0-brane in the toy model. It was also mentioned in [11] and argued in [12] that the result should also hold with coupled dilaton and general brane dimensionality $p$. Indeed, here we find that the constraint equation evaluated with (32) implies

$$
\left.\left[\lambda V c_{3}^{p-6} e^{\phi(p / 4-1 / 2)}+\Delta^{1 / 2}(p-7)(p-8)\right]\right|_{t=0}=0,
$$

thus making (32) inconsistent. As a result, there are no smooth time-reversal invariant solutions realizing S-branes in the coupled tachyon matter-supergravity system with $I S O(p+1) \times S O(8-p, 1)$ symmetry.

\section{A.2 A singularity theorem for $p<8$}

Here we present a straightforward generalization of the singularity theorem of sections (2.3), (2.4) applicable for $p<8$. As before, we will show that the average Hubble parameter $H$ given by (18) will diverge in finite time, provided the initial condition (21) holds.

Consider the general linear combination

$$
\begin{gathered}
k_{1} \times(11.1)+k_{2} \times(11.2)+k_{3} \times(11.3) \\
\Uparrow \\
L H S=R H S,
\end{gathered}
$$

where $k_{i}$ are some (for now arbitrary!) constants and the additional index $i$ in (11.i) refers to the $i$-th equation from the top in (11). We want to rewrite the resulting equation (34) so that it involves only derivatives of $H$ as defined in (18). It is easy to see that the vanishing of $\dot{H}_{\perp}$ (after eliminating $\dot{H}_{\|}$) requires

$$
k_{3}=\frac{8-p}{p+1} k_{2} .
$$


Now, the left hand side $L H S$ of (34) can be combined as

$$
\begin{aligned}
L H S= & -9 \frac{k_{1}(p+1)-k_{2}}{p+1} \dot{H}-9 \frac{k_{1}(p+1)-9 k_{2}}{p+1} H^{2} \\
& -\frac{k_{2}(p-7)(p-8)}{a_{\perp}^{2}(p+1)}-\frac{k_{1}(p+1)(8-p)}{9}\left(H_{\|}-H_{\perp}\right)^{2} .
\end{aligned}
$$

The right hand side of (34) reads

$$
\begin{aligned}
R H S= & \frac{k_{1}(7-p)+k_{2}}{16} e^{a \phi} A^{2}+\frac{k_{1}}{2}(\dot{\phi})^{2} \\
& +\frac{\left(k_{1}(p+1)-k_{2}\right) e^{(p-4) \phi / 4}}{16 \sqrt{\Delta}} V \lambda a_{\perp}^{p-8}(\dot{T})^{2} \\
& +\frac{\left(k_{1}(p+1)(6-p)+k_{2}(10+p)\right) e^{(p-2) \phi / 4}}{16 \sqrt{\Delta}(p+1)} V \lambda a_{\perp}^{p-8} .
\end{aligned}
$$

The equation (34) takes the form

$$
-9 \frac{k_{1}(p+1)-k_{2}}{p+1} \dot{H}=9 \frac{k_{1}(p+1)-9 k_{2}}{p+1} H^{2}+[\text { smth }],
$$

where [smth] can be easily deduced from (36) and (37). We will be interested in the following conditions on the $k_{i}$

$$
\begin{array}{ll}
k_{1}>0, & k_{2}>0, \\
k_{1}(p+1)-k_{2}>0, & k_{1}(p+1)-9 k_{2} \geq 0 \\
k_{1}(7-p)+k_{2} \geq 0, & k_{1}(p+1)(6-p)+k_{2}(10+p) \geq 0 .
\end{array}
$$

Note that we can always find $k_{1}, k_{2}$ satisfying (39) (notice that some conditions are $\geq$ and some other are $>$ - this is important!) provided $p<8$. Now, given (39), [smth] in (38) is always nonnegavite. So if the initial conditions for the universe are such that $\left.H\right|_{t \rightarrow-\infty}<0$, we can replace (38) with

$$
-9 \frac{k_{1}(p+1)-k_{2}}{p+1} \dot{H}=9 \frac{k_{1}(p+1)-9 k_{2}}{p+1} H^{2} .
$$

Again, the point is that if $H$ satisfying (40) will diverge in finite time, $H$ satisfying (38) will diverge earlier. It is trivial to solve (40) and see the divergence

$$
H=\frac{k_{1}(p+1)-k_{2}}{t\left(k_{1}(p+1)-9 k_{2}\right)+\delta},
$$

where $\delta$ is an integration constant. 


\section{A.3 Comparison with Leblond-Peet.}

The main result of this note is in apparent contradiction with the recent claim of the construction of singularity-free S-brane solutions in supergravity for the maximally symmetric ansatz of [11] made by Leblond and Peet in [13]. In this section we attempt to understand this contradiction.

From the analytic side, we have been able to trace back the discrepancy to what we believe is an incorrect implementation of the smearing. As we have explained in the text, the only smearing consistent with the symmetries is uniformly in the transverse space, but constant in the parallel directions. In particular, $d\left(\rho_{0}\right) / d t=0$, where $\rho_{0}$ is as in (10). This condition is not properly implemented in [13], as is apparent from their equation $(3.30)^{10}$. It appears that while some of the equations (3.31)-(3.42) do satisfy this condition, others do not, and this is one reason that their system of equations is inconsistent. For example, if $\rho_{\perp}$ is allowed to depend on the parallel directions, there should be a term containing $\partial_{\mu} \rho_{\perp}$ in the tachyon equation (3.23), and then a corresponding term later in (3.35). However, it is quite obvious that there is no way of making $\rho_{\perp}$ depend on time in a way that renders the resulting equations consistent. A time dependent $\rho_{\perp}$ will simply not satisfy the energy conservation condition (13). (One can also view this as conservation of the number of unstable branes.) To be fair, we wish to acknowledge that the corresponding equation in [11] (eq. (10)), is somewhat imprecise. The precise meaning of this equation, which is also the meaning used in the equations of motion and singularity analysis of [11], is as in eqs. (3) and (10) of the present note.

Note that in (29), the Maxwell equation can be explicitly integrated, to give

$$
A=\frac{Q+\lambda F(T)}{e^{a \phi} c_{3}^{8-p}},
$$

where $F=\int f$, and $Q$ is an integration constant. Eq. (42) has the desirable physical interpretation that the total "charge of the S-brane" (the asymptotic value of the RRfield) can be completely determined from the initial conditions on the tachyon. One of the consequences of the time-dependence of $\rho_{\perp}$ in [13] is that the Maxwell equation can not be integrated anymore in this way.

For completeness, we now write the equations in string frame. The transformation

\footnotetext{
${ }^{10} \mathrm{We}$ are referring to the numbering of equations in hep-th/0303035v1.
} 
to string frame in the notation of [13] is

$$
c_{1}=e^{-\phi / 4}, \quad c_{2}=a e^{-\phi / 4}, \quad c_{3}=R e^{-\phi / 4}, \quad A=c_{1}^{-1} c_{2}^{-p-1} \dot{C} .
$$

Eq.(3.31) of [13] becomes

$$
\ddot{C}+\dot{C}\left[(8-p) \frac{\dot{R}}{R}-(p+1) \frac{\dot{a}}{a}\right]=\lambda a^{p+1} R^{p-8} f(T) \dot{T} .
$$

Eq.(3.34) of [13] becomes

$$
\begin{aligned}
& \ddot{\Phi}+\dot{\Phi}\left[(8-p) \frac{\dot{R}}{R}+(p+1) \frac{\dot{a}}{a}\right]-2 \dot{\Phi}^{2} \\
& =\frac{(3-p)}{4}\left(\frac{e^{\Phi} \dot{C}}{a^{(p+1)}}\right)^{2}+\frac{\lambda}{4} e^{\Phi} V(T) R^{p-8}\left[(3-p) \sqrt{\Delta}-\frac{1}{\sqrt{\Delta}}\right]
\end{aligned}
$$

Eq.(3.35) of [13] becomes

$$
\ddot{T}=\Delta\left\{\dot{\Phi} \dot{T}-\dot{T}\left[(p+1) \frac{\dot{a}}{a}\right]-\frac{1}{V(T)} \frac{d V(T)}{d T}-\frac{f(T)}{V(T)} \dot{C} e^{\Phi} a^{-(p+1)} \sqrt{\Delta}\right\},
$$

Note the sign typo for $f / V$ term. Finally, the Einstein equations in [13] (3.40)-(3.42) are correct provided one makes the by now familiar replacement

$$
\lambda \rightarrow \lambda R^{p-8}
$$

We have explicitly verified that the equations of [13] modified as above are consistent. All conclusions we have reached in the main text about singularities in the geometry obviously hold here as well.

Finally, let us comment that the numerical integration of the equations in [13] is rather problematic. Since the equations are inconsistent, one is bound to get different results depending on what subset of equations one chooses to numerically integrate. In any case, it also appears [29] that the code used in [13] is unstable and this appears to be another possible reason for the contradiction.

\section{References}

[1] M. Gutperle and A. Strominger, "Spacelike branes," JHEP 0204, 018 (2002) [arXiv:hep-th/0202210]. 
[2] A. Sen "Rolling tachyon," JHEP 0204, 048 (2002) [arXiv:hep-th/0203211]; "Tachyon matter," arXiv:hep-th/0203265; "Field theory of tachyon matter," arXiv:hep-th/0204143.

[3] C. M. Chen, D. V. Gal'tsov and M. Gutperle, "S-brane solutions in supergravity theories," Phys. Rev. D 66, 024043 (2002) [arXiv:hep-th/0204071].

[4] M. Kruczenski, R. C. Myers and A. W. Peet, "Supergravity S-branes," JHEP 0205, 039 (2002) [arXiv:hep-th/0204144].

[5] S. Roy, "On supergravity solutions of space-like Dp-branes," JHEP 0208, 025 (2002) [arXiv:hep-th/0205198].

[6] N. S. Deger and A. Kaya, "Intersecting S-brane solutions of D = 11 supergravity," JHEP 0207, 038 (2002) [arXiv:hep-th/0206057];

[7] K. Ohta and T. Yokono, "Gravitational approach to tachyon matter," Phys. Rev. D 66, 125009 (2002) [arXiv:hep-th/0207004].

[8] N. Ohta, "Intersection rules for S-branes," Phys. Lett. B 558, 213 (2003) [arXiv:hep-th/0301095].

[9] N. Ohta, "Null-brane solutions in supergravities," Phys. Lett. B 559, 270 (2003) [arXiv:hep-th/0302140].

[10] N. S. Deger, "Non-standard intersections of S-branes in D = 11 supergravity," JHEP 0304, 034 (2003) [arXiv:hep-th/0303232].

[11] A. Buchel, P. Langfelder and J. Walcher, "Does the tachyon matter?," Annals Phys. 302, 78 (2002) [arXiv:hep-th/0207235].

[12] A. Buchel and J. Walcher, "The tachyon does matter," arXiv:hep-th/0212150.

[13] F. Leblond and A. W. Peet, "SD-brane gravity fields and rolling tachyons," JHEP 04, 048 (2003) [arXiv:hep-th/0303035].

[14] A. Strominger, "Open string creation by S-branes," arXiv:hep-th/0209090.

[15] A. Sen, "Time and tachyon," arXiv:hep-th/0209122. 
[16] M. Gutperle and A. Strominger, "Timelike boundary Liouville theory," arXiv:hep-th/0301038.

[17] A. Maloney, A. Strominger and X. Yin, "S-brane thermodynamics," arXiv:hep-th/0302146.

[18] T. Okuda and S. Sugimoto, "Coupling of rolling tachyon to closed strings," Nucl. Phys. B 647, 101 (2002) [arXiv:hep-th/0208196].

[19] B. Chen, M. Li and F. L. Lin, "Gravitational radiation of rolling tachyon," JHEP 0211, 050 (2002) [arXiv:hep-th/0209222].

[20] N. Lambert, H. Liu and J. Maldacena, "Closed strings from decaying D-branes," arXiv:hep-th/0303139.

[21] S. Sugimoto and S. Terashima, "Tachyon matter in boundary string field theory," JHEP 0207, 025 (2002) [arXiv:hep-th/0205085].

[22] K. Hashimoto, P. M. Ho and J. E. Wang, "S-brane actions," arXiv:hep-th/0211090.

[23] F. Larsen, A. Naqvi and S. Terashima, "Rolling tachyons and decaying branes," JHEP 0302, 039 (2003) [arXiv:hep-th/0212248].

[24] D. Kutasov and V. Niarchos, "Tachyon effective actions in open string theory," arXiv:hep-th/0304045.

[25] S. W. Hawking and G. F. R. Ellis, "The large scale structure of space-time," Cambridge Univ. Press, London, 1973.

[26] P. Di Vecchia, M. Frau, I. Pesando, S. Sciuto, A. Lerda and R. Russo, "Classical p-branes from boundary state," Nucl. Phys. B 507, 259 (1997) [arXiv:hep-th/9707068].

[27] M. R. Garousi, "Tachyon couplings on non-BPS D-branes and Dirac-Born-Infeld action," Nucl. Phys. B 584, 284 (2000) [arXiv:hep-th/0003122].

[28] Work in progress.

[29] F. Leblond and A. W. Peet, "A note on the singularity theorem for supergravity SD-branes," arXiv:hep-th/0305059. 